Conclusions MS is highly prevalent in Kazak populations in Xinjiang, particularly among men. And there was a tendency of increase in IMTwith increasing components of metabolic syndrome. Metabolic syndrome in Kazak populations has adverse effect on early atherosclerosis. To find out high-risk MS groups is an important measure for preventing early atherosclerosis.

\section{e0248 INFLUENCE OF GENDER ON PULSE WAVE VELOCITY AND ARTERIAL COMPLIANCE IN PATIENTS WITH HYPERTENSION AND CORONARY HEART DISEASE 1}

doi:10.1136/hrt.2010.208967.248

Gao Hai-qing, Li Bao-ying, Li Xiao-Li, Cheng Mei, Yu Fei. Qi-Lu Hospital of Shandong University

Objective The lower incidence of atherosclerosis in premenopausal women than in men was an established epidemiological observation. However, the incidence of cardiovascular disease in women increased dramatically after postmenopausal years. Compliance and distensibility are wall properties of arteries, which may play a role in cardiovascular disease. The purpose of this study was to investigate whether the influence of gender on arterial compliance in patients with hypertension (HP) and/or coronary heart disease (CHD).

Methods The cohort consisted of 500 patients with HP and/or $\mathrm{CHD}$ who were registered as outpatients in the Department of Geriatrics of the Qi-Lu Hospital of Shandong University from February 2007 to April 2008 (age from 45 to 87 year). All subjects gave informed written consent. The study protocol was approved by the Ethics Board of Qi-Lu Hospital of Shandong University. Five hundred patients (330 men, 170 women) were divided into four groups: premenopausal women $(n=54)$ and age-matched normotensive men $(n=118)$, postmenopausal women $(n=116)$ and agematched men $(n=212)$. Postmenopausal women did not receive hormone replacement therapy. Carotid-femoral pulse wave velocity (CF-PWV), capacitive arterial compliance (C1), and oscillatory arterial compliance (C2) were measured using Complior SP automatic device and DO-2020 cardiovascular profiling instrument. Premenopausal and postmenopausal women were compared with age-matched men.

Results Postmenopausal women had lower positive rates of CFPWV and C1 than age-matched men $(44.83 \%$ vs $72.17 \%, \mathrm{p}<0.01$; $25.86 \%$ vs $41.04 \%, p<0.01$ ), whereas the positive rates of $\mathrm{C} 2$ was higher in postmenopausal women $(35.34 \%$ vs $15.57 \%, \mathrm{p}<0.01)$. But the positive rates of CF-PWV, C1 and C2 of premenopausal women did not differ significantly than age-matched men $(p>0.05)$. Women with CHD, HP+CHD had lower CF-PWV $(p<0.05)$ than men. CF-PWV of women with HP did not differ significantly than men with HP ( $p>0.05)$. Moreover, women with $\mathrm{CHD}, \mathrm{HP}+\mathrm{CHD}$ had lower positive rates of CF-PWV and C1 than men $(3.33 \%$ vs $36.54 \%, 3.33 \%$ vs $21.15 \%, p<0.05 ; 58.11 \%$ vs $81.38 \%, 29.73 \%$ vs $50.34 \%, p<0.01)$, whereas the positive rates of C2 was higher in women with CHD, HP, HP+CHD (30.00\% vs $9.62 \%, 22.73 \%$ vs $9.77 \%, 32.43 \%$ vs $18.62 \%, p<0.05)$. But the positive rates of CF-PWV of women with HP did not differ significantly than men with HP ( $p>0.05)$. In multiple regression analysis of patients with CHD, HP and CHD, CF-PWV, C1 and C2 were significantly correlated with gender (women) $(\mathrm{r}=0.480$, $-0.259,-0.242, \mathrm{p}<0.05 ; 0.200,-0.145,-0.237, \mathrm{p}<0.05)$, whereas there were no close correlations between CF-PWV and gender (women) in the patients with HP ( $\mathrm{r}=0.095, \mathrm{p}>0.05)$.

Conclusion The effect of cardiovascular risk factors on arterial compliance was not uniform but depends on gender and menopause.

\section{e0249 PROGNOSTIC VALUE OF PREMATURE VENTRICULAR CONTRACTION ORIGINATING FROM DIFFERENT LOCATION FOR PATIENTS WITH CHRONIC HEART FAILURE}

doi:10.1136/hrt.2010.208967.249

Yu Shengbo, Zhao Qingyan, Huang He, Chen Donge, Qin Mu, Huang Congxin. Renmin Hospital of Wuhan University

Background Several studies have suggested that ventricular arrhythmias detected by ambulatory ECG (AECG) may identify patients with chronic heart failure (CHF) at high risk for cardiovascular death. However, few studies aim at whether different origins of premature ventricular contraction (PVCs) can hold any prognostic value in CHF patients.

Methods Ventricular arrhythmias were analysed and quantified by use of prespecified criteria on baseline AECG from 206 patients diagnosed with CHF and left ventricular ejection fraction (LVEF) $\leq 40 \%$. After follow-up, the patients were divided into two groups: survival group and cardiac death group by an independent group. The relationship between cardiovascular mortality and AECG parameters were determined respectively by univariate and multivariate analyses.

Results 206 patients were enrolled and 198 (96.12\%) patients were completed in this study. After follow up, there were 37 deaths. A-V block, 24 h-average heart rate, number of PVCs, presence of nonsustained ventricular tachycardia (NSVT), frequency of NSVT episodes, fastest rate of NSVT, PVCs originating from left ventricular inflow tract (LVIT), NSVT originating from LVIT and LOWN grade were univariate predictors of cardiovascular mortality. In multivariate logistic regression analysis, $24 \mathrm{~h}$-average heart rate, number of PVCs, PVCs originating from LVIT and frequency of NSVT episodes were significant independent predictors of cardiovascular mortality.

Conclusion Determinating the location of PVCs by AECG can indicate prognosis of CHF patients.

\section{e0250 STUDY AUGMENTATION INDEX AND INFLUENTIAL FACTORS IN UYGUR HEALTH ADULTS IN HETIAN, XINJIANG}

doi:10.1136/hrt.2010.208967.250

Liang Xiaohui, Xu Xinjuan, Ma Yanping, Sun Lihua, Ma Yitong, Huang Ding, Yang Yining. First Affiliated Hospital, Xinjiang Medical University, Urumqi, China

Objective To investigate augmentation index (AIx) and the affecting factors in Xinjiang Uygur health adults.

Methods The total of 2366 Uygur natural adults were investigated. 559 health adults (male 251, female 308) were selected in the study and the mean age was $(44.95 \pm 11.87)$ years. All the subjects were measured the following indexes: questionnaire, physical examination, fasting blood glucose, blood lipid, renal function and C-reactive protein (CRP). Central systolic blood pressure (cSBP) and central augmentation index (AIx) were measured by Sphygmocor.

Results AIx in female was higher than in male (male: 18.52 \pm 11.27 , female: $29.84 \pm 8.44, p<0.05)$. The same result was found in different age groups. Unadjusted correlation analysis showed AIx was positively related to age in both genders (male: $r=0.548$, female $=0.405, \mathrm{p}<0.05$ ), as well as adjusted other influential factors (male: $\mathrm{r}=0.182$, female $=0.234, \mathrm{p}<0.05$ ). Multivariate regression analysis showed that cSBP, systolic blood pressure (SBP), age, weight and CRP were main factors which affected AIx in male, while age, cSBP, SBP, height and CRP were main factors which affected AIx in female.

Conclusion AIx in female was higher than in male. AIx was positively related to age in both genders. Age, cSBP, SBP and CRP were main factors affected AIx in Xinjiang Uygur health adults. 